\title{
IceCube/DeepCore Results and PINGU
}

\author{
Thomas Ehrhardt* for the IceCube-Gen2 Collaboration ${ }^{\dagger}$ \\ Universität Mainz, Germany \\ E-mail: tehrhardteicecube.wisc.edu
}

\begin{abstract}
As the low-energy infill array of the IceCube Neutrino Observatory at the South Pole, DeepCore allows performing high statistics studies of atmospheric neutrinos with energies down to around $5 \mathrm{GeV}$. This low energy threshold enables DeepCore to resolve characteristic distortions of the atmospheric neutrinos' energy and zenith angle distributions. Based on a set of neutrinos collected over three years of operation, the atmospheric oscillation parameters $\theta_{23}$ and $\Delta m_{32}^{2}$ are measured with a precision competitive with long-baseline experiments. With a further reduction of the inice energy threshold and improved reconstruction resolutions, the "IceCube Upgrade" will focus on dedicated oscillation measurements that are unique to the flux of atmospheric neutrinos. At the same time, the upgrade will serve as a stepping stone toward the physics goals of PINGUan envisaged, even more densely instrumented array dedicated to determining the neutrino mass ordering.
\end{abstract}

The 19th International Workshop on Neutrinos from Accelerators-NUFACT2017

25-30 September, 2017

Uppsala University, Uppsala, Sweden

\footnotetext{
*Speaker.

${ }^{\dagger}$ see http://icecube.wisc.edu/collaboration/authors/icecubegen2_2017 for author list
} 


\section{Introduction}

In the three-flavour neutrino oscillation scenario, mass and flavour eigenstates of neutrinos are connected by the unitary $3 \times 3$ PMNS mixing matrix [1, 2], which transforms between the two eigenbases. Oscillations - first clear evidence of which was found by Super-Kamiokande in 1998 [3] — are the result of both mixing of the states and their quantum mechanical interference. After having propagated over a distance $L$ (the baseline), a neutrino produced in a certain flavour eigenstate will exhibit a non-vanishing probability of being detected in a different flavour eigenstate. As of today, only the octant of the "atmospheric" mixing angle $\theta_{23}$, the value of the CPviolating phase $\delta_{\mathrm{CP}}$, and the neutrino mass ordering (NMO, i.e. the sign of $\Delta m_{32}^{2}$ ), are still largely unknown $[4,5]$.

The megaton-scale DeepCore infill of the IceCube detector [6,7] has proven to be a valuable tool in studying atmospheric neutrinos at $\mathrm{GeV}$ energies, whose oscillations proceed over baselines ranging up to the Earth's diameter of about $12700 \mathrm{~km}$. The energy range not only allows for a precision measurement of the first survival probability minimum of upgoing muon neutrinos at around $25 \mathrm{GeV}$, but also enables establishing the rate of tau neutrino appearance and thereby searching for possible physics beyond the standard three-flavour oscillation paradigm.

This document discusses a recent measurement of standard atmospheric neutrino oscillations with IceCube DeepCore. It also lays out the case for future upgrades to IceCube, which will be able to significantly extend the latter's capabilities in terms of fundamental neutrino physics, such as the question of the origin of astrophysical neutrinos, or the NMO.

\section{The IceCube detector}

Instrumenting a total volume of $1 \mathrm{~km}^{3}$ of glacial ice, the IceCube Neutrino Observatory [6] is a huge neutrino detector constructed at the location of the Amundsen-Scott South Pole Station in Antarctica. At depths between $1450 \mathrm{~m}$ and $2450 \mathrm{~m}$, more than 5000 digital optical modules (DOMs) distributed on 86 strings observe the Cherenkov light emitted by charged particles in their vicinity, produced when neutrinos interact in the ice. As shown in Fig. 1, eight of the innermost strings are more densely packed, with inter-string spacings ranging from around $40 \mathrm{~m}$ up to $70 \mathrm{~m}$, compared to a typical spacing of $125 \mathrm{~m}$ elsewhere in IceCube. These strings comprise the DeepCore infill array [7].

While IceCube was originally designed to detect high-energy neutrinos of astrophysical origin in the $\mathrm{TeV}$ to $\mathrm{PeV}$ regime - a goal that was accomplished in 2014 [8] - its DeepCore extension reduces IceCube's energy threshold by two orders of magnitude down to a few GeV. As a result, IceCube has proven to be able to make competitive measurements of atmospheric neutrino oscillations [9].

\section{A new, high-statistics measurement of neutrino oscillations with DeepCore}

The Cherenkov light pattern of neutrino interactions in IceCube can be classified according to two different topologies. Light from hadronic and electromagnetic showers is emitted within the immediate vicinity $(\mathscr{O}(\mathrm{m}))$ of the neutrino interaction vertex, and the observed light distribution is 


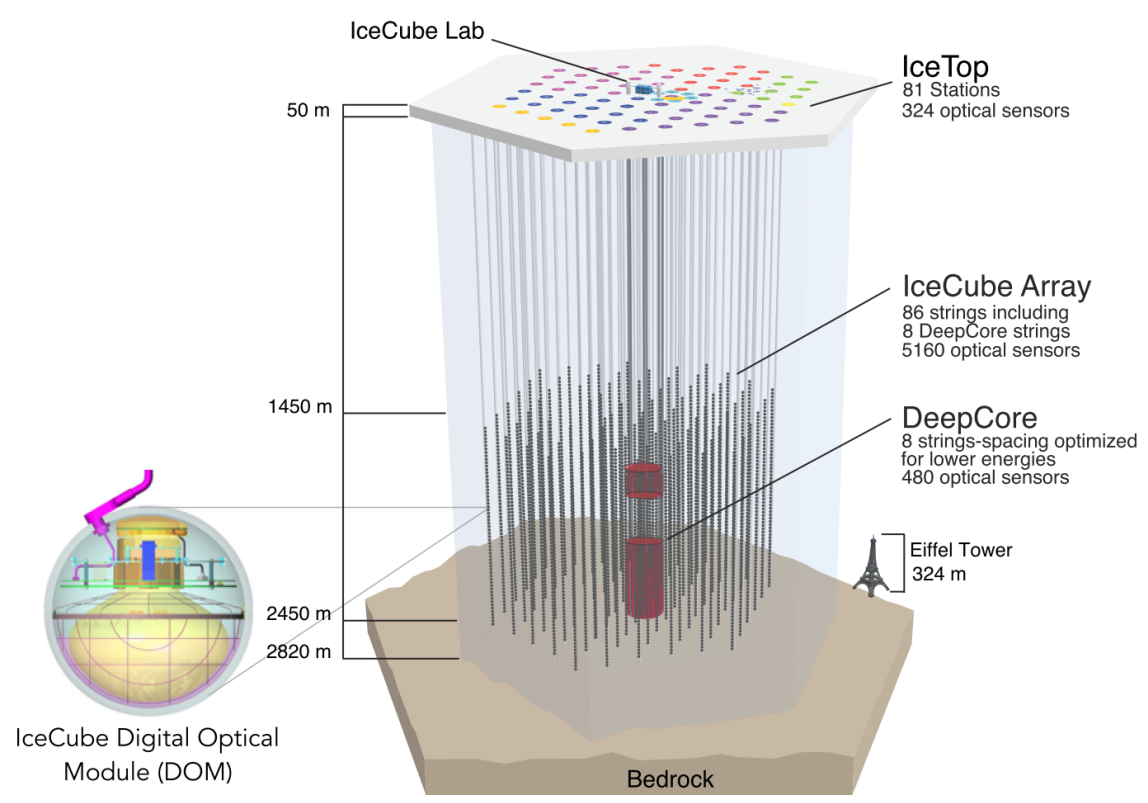

Figure 1: The IceCube Neutrino Observatory—with its low-energy infill array DeepCore-comprising 5160 optical modules. As shown by the sketch on the left, each module (DOM) consists of a glass pressure sphere enclosing a downward-facing $10^{\prime \prime}$ PMT.

roughly spherical. Events of this type are referred to as "cascade-like". In case the interaction produces a muon, however, the event is considered "track-like". For atmospheric neutrino oscillation studies, the track-like sample is predominantly composed of charged current (CC) muon neutrino interactions, thus bearing the signature of muon neutrino disappearance with amplitude and oscillation length crucially determined by the atmospheric oscillation parameters $\theta_{23}$ and $\Delta m_{32}^{2}$, respectively. The cascade-like sample, on the other hand, is a more uniform mixture of mis-identified $\mathrm{CC}$ interactions of muon neutrinos and of all other remaining neutrino flavours, and as such still somewhat sensitive to the oscillations.

In order to measure the atmospheric oscillation parameters, more than 41000 up- and downgoing atmospheric neutrino events from three years (2012-2015) of operation of IceCube DeepCore are classified as cascade- or track-like, with reconstructed energies $E$ between $5.6 \mathrm{GeV}$ and $56 \mathrm{GeV}$. The fit is performed in $2 \mathrm{D}$ bins of energy $E$ and $\cos \theta_{z}$, the reconstructed zenith angle of the neutrino, which can be directly translated into a corresponding oscillation baseline $L$. Sources of systematic uncertainties taken into account are the atmospheric neutrino flux, both as a function of energy and zenith, the neutrino interaction cross section, the efficiency of rejecting atmospheric muons, as well as the optical efficiency of the DOMs.

The left panel of Fig. 2 shows the resulting best fit event distributions-broken down into the different neutrino flavours and interaction types - as well as the data in both event classes, projected onto the observed ratio $L / E$. As can be seen from a comparison to the no-oscillation expectation, oscillations manifest mainly through a deficit in the observed event rates for $L / E \gtrsim 100 \mathrm{~km} \mathrm{GeV}^{-1}$, which is more pronounced in the track-like channel.

The confidence region of the fit for $\sin ^{2} \theta_{23}$ and $\Delta m_{32}^{2}$ is depicted in the right panel of Fig. 2, where the best fit values and uncertainties are $\sin ^{2} \theta_{23}=0.51_{-0.09}^{+0.07}, \Delta m_{32}^{2}=2.31_{-0.13}^{+0.11} \times 10^{-3} \mathrm{eV}^{2}$ 

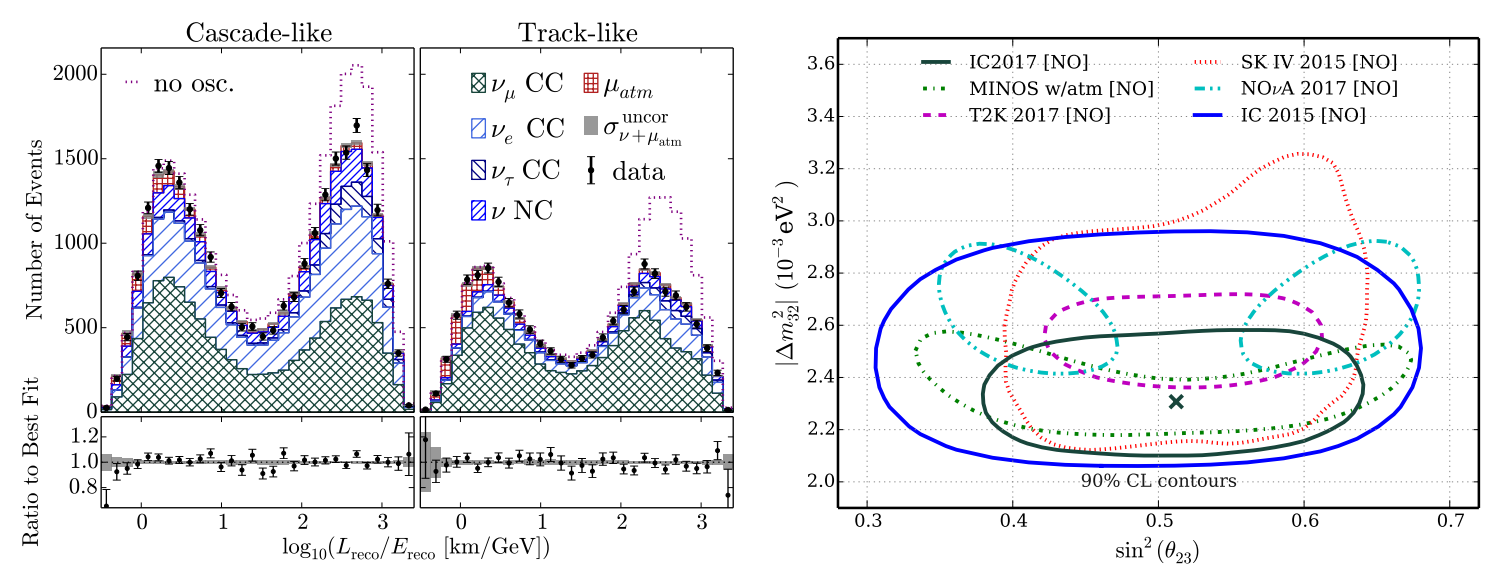

Figure 2: Left panel: $L / E$ distributions of cascade-like and track-like events from data and the best fit for a new three-year measurement of atmospheric oscillations with IceCube DeepCore. Right panel: Region allowed at $90 \%$ C.L. for the parameters $\sin ^{2} \theta_{23}$ and $\Delta m_{32}^{2}$ (solid black line), assuming the normal ordering. The cross indicates the best fit point. This result is compared to an earlier IceCube analysis $[9,15]$ (solid blue), and to measurements obtained by other experiments [11, 12, 13, 14] (various dashed and dotted lines).

in the normal ordering. In order to ensure its claimed coverage, the $90 \%$ C.L. contour is obtained under the approach of Feldman and Cousins [10]. These results are consistent with the most recent ones reported by other atmospheric and accelerator neutrino experiments [11, 12, 13, 14], and make use of a neutrino data set about one order of magnitude higher in energy, which in addition is subject to a very distinct set of systematic uncertainties. The figure also shows by how much the analysis at hand improves upon an earlier one based on an order of magnitude less statistics [9, 15]. Further details of the analysis presented here are provided in Ref. [16].

A search for tau neutrino appearance has been performed on the same set of data. It is expected to yield a sensitivity on the normalisation of the tau neutrino appearance rate of around $30 \%$ if considering charged and neutral current interactions simultaneously.

\section{Looking to the future with the IceCube Upgrade and PINGU}

Building on the experience gained with IceCube, the IceCube upgrade proposal and the envisaged PINGU extension seek to extend the capabilities of IceCube toward a precision measurement of atmospheric oscillations, physics beyond the Standard Model, as well as the detection of cosmic neutrinos. A brief discussion of both projects is provided in the following.

With only seven additional strings of photosensors-deployed deep in the central region of DeepCore, where the ice is clearest - the proposed "IceCube Upgrade" will lead to a significant augmentation of the detector's low-energy neutrino physics portfolio. Taken together, a string separation well below the effective photon scattering and absorption lengths of the ice and a reduced vertical spacing between multi-PMT optical modules (mDOMs) will greatly benefit reconstruction and flavour identification in the $\mathrm{GeV}$ energy regime. A suite of novel calibration devices will be deployed in order to further the modelling of the ice's optical properties. This in turn will be conducive to all oscillation analyses as well as to high-energy neutrino astronomy, even retroactively. In the case of the latter, ice properties are the major systematic source of uncertainty contributing to 


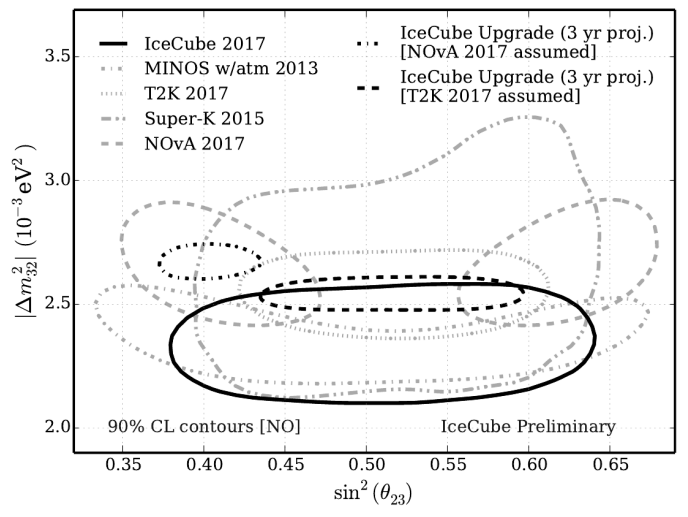

Figure 3: Projected sensitivities to atmospheric oscillation parameters using three years of data from the IceCube Upgrade. The assumed true values correspond to the NOvA 2017 first-octant [13] and the T2K 2017 [14] best fits. Existing measurements are the same as those displayed in Fig. 2.

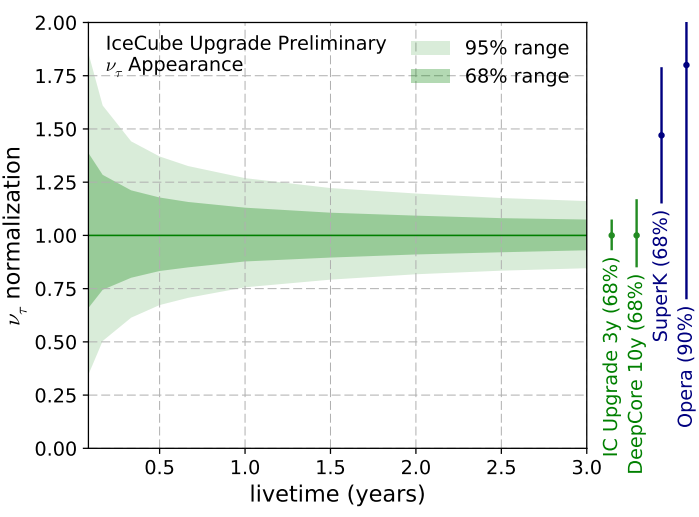

Figure 4: Projected sensitivity of the IceCube Upgrade to the tau neutrino normalisation as a function of exposure time, compared to current measurements [17, 18] and a 10-year DeepCore projection.

the angular resolution of identified astrophysical neutrino cascade events and simultaneously limit the efficiency of atmospheric background vetoing techniques.

In the atmospheric neutrino sector, the IceCube Upgrade is expected to lead to a considerable increase of the precision of the measurement of $\sin ^{2} \theta_{23}, \Delta m_{32}^{2}$, and the tau neutrino appearance rate (normalisation), as summarised by Fig. 3 and Fig. 4. With the same event selection and analysis techniques as employed at present, a tau neutrino normalisation precision of better than $10 \%$ will be achieved after only three years with the upgrade in place. Also, the projected sensitivity to the octant of $\theta_{23}$ exceeds $3 \sigma$ for NOvA's best fit in the first octant.

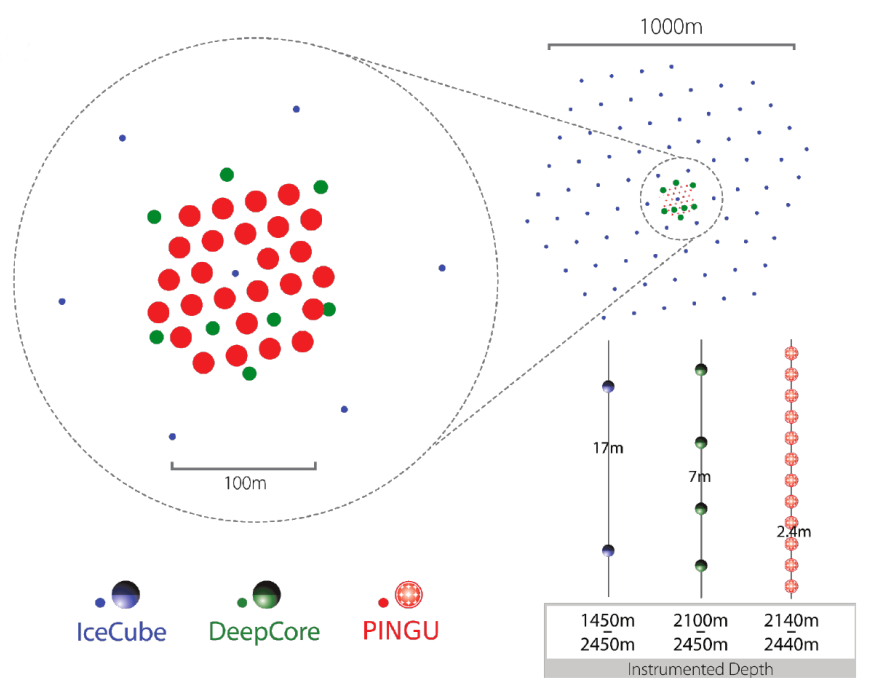

Figure 5: Top view of PINGU (red circles), its location within the DeepCore array (green), and the whole IceCube detector (blue). The area of the circles is representative of the instrumented photocathode density on a given string. A comparison of the vertical photosensor spacing is provided on the lower right. 

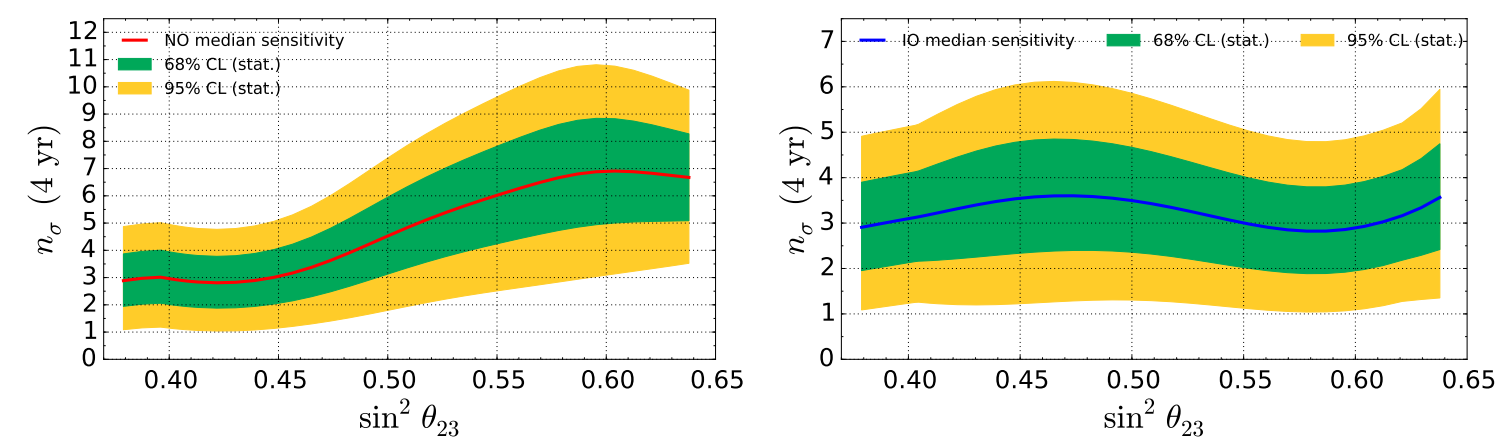

Figure 6: PINGU median sensitivity to the NMO after four years of exposure time as a function of the true value of $\sin ^{2} \theta_{23}$. The left panel shows the case of true normal ordering, while the right panel assumes the inverted ordering to be true.

PINGU $[19,20]$ will take the concept of the IceCube Upgrade even further and add 19 extra strings equipped with mDOMs to fill in the deepest region of DeepCore as can be seen in Fig. 5. As a result, PINGU will have increased sensitivity and resolution in the energy range between around $5 \mathrm{GeV}$ and $15 \mathrm{GeV}$, which-as it is heavily impacted by matter effects-is key to resolving the NMO as well as the value of $\theta_{23}$. Matter effects will impact oscillations of neutrinos (anti-neutrinos) if the NMO is normal (inverted). Even though the oscillation probabilities are nearly symmetric between neutrinos and anti-neutrinos when switching the NMO, and despite the absence of event-by-event neutrino vs. anti-neutrino discrimination in PINGU, the NMO will leave a net imprint due to differences in the respective atmospheric fluxes and the interaction cross sections [21].

Following through with a similar analysis approach as in the case of the muon neutrino disappearance analysis with DeepCore, distinctive asymmetries in the $E \times \cos \theta_{z}$ spectra of cascade- and track-like events can be exploited in order to determine the NMO. PINGU's projected sensitivity is displayed in Fig. 6 for the two possible true realisations of the NMO. A $\gtrsim 3 \sigma$ C.L. measurement can be expected for just beyond four years of operation, regardless of the mixing angle $\theta_{23}$.

Apart from its unique ability to resolve the NMO across the whole range of $\sin ^{2} \theta_{23}$ values, which in addition is only very weakly impacted by $\delta_{\mathrm{CP}}$, PINGU will improve upon the oscillation physics potential of the IceCube Upgrade across the board. As an example, the tau neutrino appearance rate will be constrained to better than $10 \%$ with only a single year of data, and a $5 \sigma$ exclusion of no appearance given standard oscillations will be in reach within just one month of data taking. A detailed and up-to-date discussion of PINGU can be found in Ref. [20].

\section{Conclusion}

IceCube and its DeepCore infill keep pushing their sensitivity to the atmospheric neutrino oscillation parameters by virtue of improved analyses and increasing statistics. The precision to which $\sin ^{2} \theta_{23}$ and $\Delta m_{32}^{2}$ are determined using a data set of more than 41000 atmospheric neutrinos collected over the course of three years of operation is competitive to that of accelerator based experiments. Complementarity ensues from the fact that this analysis is performed at significantly higher neutrino energies, and is impacted by an almost orthogonal set of systematic uncertain- 
ties. Follow-up studies of atmospheric neutrinos are underway, looking to further improve control over systematic uncertainties and thereby extend the low-energy oscillation physics potential of IceCube.

Future extensions of the current detector hold in store an even more multifaceted physics reach. The proposed IceCube Upgrade will allow for a measurement of the tau neutrino appearance rate with a precision of better than $10 \%$, and put to test the unitarity of the tau sector of the PMNS matrix. Dedicated advanced detector calibration devices deployed with the proposed upgrade will be conducive both to ongoing IceCube oscillation studies as well as to observations of astrophysical neutrinos at the highest energies. Looking beyond the IceCube Upgrade, PINGU plays a key role in determining the neutrino mass ordering, and will provide greatly enhanced precision measurements in the atmospheric oscillation sector.

\section{References}

[1] B. Pontecorvo, Sov. Phys. JETP 6, 429 (1957)

[2] Z. Maki, M. Nakagawa and S. Sakata, Prog. Theor. Phys. 28, 870 (1962)

[3] Y. Fukuda et al. (Super-Kamiokande Collaboration), Phys. Rev. Lett. 81, 1562 (1998)

[4] I. Esteban, M. C. Gonzalez-Garcia, M. Maltoni, I. Martinez-Soler and T. Schwetz, JHEP 01, 87 (2017)

[5] I. Esteban, M. C. Gonzalez-Garcia, A. Hernandez-Cabezudo, M. Maltoni, I. Martinez-Soler and T. Schwetz, NuFIT 3.1 (2017), http://www.nu-fit.org

[6] M. G. Aartsen et al. (IceCube), JINST 12, P03012 (2017)

[7] R. Abbasi et al. (IceCube), Astropart. Phys. 35, 615 (2012)

[8] M. G. Aartsen et al. (IceCube), Phys. Rev. Lett. 113, 101101 (2014)

[9] M. G. Aartsen et al. (IceCube), Phys. Rev. D91, 072004 (2015)

[10] G. J. Feldman and R. D. Cousins, Phys. Rev. D57, 3873 (1998)

[11] P. Adamson et al. (MINOS), Phys. Rev. Lett. 110, 251801 (2013)

[12] R. Wendell (Super-Kamiokande), AIP Conf. Proc. 1666, 100001 (2015)

[13] P. Adamson et al. (NOvA), Phys. Rev. Lett. 118, 151802 (2017)

[14] K. Abe et al. (T2K), Phys. Rev. Lett. 118, 151801 (2017)

[15] D. J. Koskinen (IceCube), Journal of Physics: Conference Series 888, 012023 (2017)

[16] M. G. Aartsen et al. (IceCube), "Measurement of Atmospheric Neutrino Oscillations at 6-56 GeV with IceCube DeepCore", arXiv:1707.07081

[17] Z. Li (Super-Kamiokande, Hyper-Kamiokande), Nucl. Part. Phys. Proc. 287-288, 147 (2017)

[18] N. Agafonova et al. (OPERA), Phys. Rev. Lett. 115, 121802 (2015)

[19] M. G. Aartsen et al. (IceCube), J. Phys. G44, 054006 (2017)

[20] M. G. Aartsen et al. (IceCube), "Letter of Intent: The Precision IceCube Next Generation Upgrade (PINGU)", arXiv:1401.2046v2 (2017)

[21] J. A. Formaggio and G. P. Zeller, Rev. Mod. Phys. 84, 1307 (2012) 